Frank T. C. Tschirch Kerstin Göpfert Johannes M. Fröhlich Genevieve Brunner Dominik Weishaupt

Received: 26 April 2006

Revised: 7 July 2006

Accepted: 25 August 2006

Published online: 9 November 2006

(C) Springer-Verlag 2006
F. T. C. Tschirch $(\bowtie) \cdot$ K. Göpfert ·

G. Brunner - D. Weishaupt Institute of Diagnostic Radiology, University Hospital Zürich,

Rämistrasse 100 ,

CH-8091 Zürich, Switzerland e-mail: Frank.Tschirch@usz.ch

Tel.: +41-1-2553060

Fax: +41-1-2554506

J. M. Fröhlich

Klus-Apotheke,

Hegibachstrasse 102,

CH-8032 Zürich, Switzerland

\section{Low-dose intranasal versus oral midazolam for routine body MRI of claustrophobic patients}

Abstract The purpose of this study was to assess prospectively the potential of low-dose intranasal midazolam compared to oral midazolam in claustrophobic patients undergoing routine body magnetic resonance imaging (MRI). Seventytwo adult claustrophobic patients referred for body MRI were randomly assigned to one of two treatment groups (TG1 and TG2). The 36 patients of TG1 received $7.5 \mathrm{mg}$ midazolam orally $15 \mathrm{~min}$ before MRI, whereas the 36 patients of TG2 received one (or, if necessary, two) pumps of a midazolam nasal spray into each nostril immediately prior to MRI (in total, 1 or $2 \mathrm{mg}$ ). Patients' tolerance, anxiety and sedation were assessed using a questionnaire and a visual analogue scale immediately before and after MRI. Image quality was evaluated using a five-point-scale. In TG1, 18/36 MRI examinations $(50 \%)$ had to be cancelled, the reduction of anxiety was insufficient in $12 / 18$ remaining patients $(67 \%)$. In TG2, 35/36 MRI examinations (97\%) were completed successfully, without relevant adverse effects. MRI image quality was rated higher among patients of TG2 compared to TG1 $(p<0.001)$. Low-dose intranasal midazolam is an effective and patientfriendly solution to overcome anxiety in claustrophobic patients in a broad spectrum of body MRI. Its anxiolytic effect is superior to that of the orally administrated form.

Keywords Midazolam .

Administration · Intranasal · Phobic disorders · Magnetic resonance imaging

\section{Introduction}

Although the architecture and design of magnets for magnetic resonance imaging (MRI) have profoundly changed over the last few years, staying enclosed in a magnet may trigger the panic reactions in patients suffering from claustrophobia. Hence, the investigation of claustrophobic patients in a MRI scanner still represents a challenge.

According to the literature, up to $10 \%$ of all MRI examinations cannot be completed, or even started, because of claustrophobia [1, 2]. Although there are some alternative techniques to facilitate the MRI of claustrophobic patients (e.g. prism eyeglasses, verbal tranquillisation), medicamentous sedation and anxiolysis remain the most useful options in order to achieve good examination results. When medicamentous sedation for MRI is desired, benzodiazepines are used in most MRI centres. These medicaments are often administered intravenously or orally, whereas the latter application is preferred. A frequently used agent is midazolam, which is approved for oral, i.v. and intramuscular use in most countries of the world.

The intranasal use of midazolam has been investigated in pediatric as well as adult populations [3-8]. Intranasal application has the following advantages compared to oral application: absent hepatic first-pass metabolisation, resulting in a faster onset of action and a higher bioavailability (of up to $83 \%$ ), minor toxicity and improved controllability due to the missing peak concentration, and a lesser sedation $[4,8,9]$. 
In a prospective double-blinded placebo controlled randomised trial, Hollenhorst et al. [2] have shown a significant reduction of MRI-related anxiety and improved MRI image quality in patients who received intranasal midazolam. In the study of Hollenhorst et al. [2], the dose of the midazolam was relatively high, and all patients were scheduled for MRI of the head or the upper body.

The purpose of this study was to assess the potential of a ready-to-use low-dose $(1 \mathrm{mg})$ midazolam nasal spray compared to the oral application of midazolam in claustrophobic patients undergoing routine MRI in a broad spectrum of MRI body investigation techniques.

\section{Patients and methods}

The study was approved by the local ethics committee and written informed consent was obtained from all of the participating patients prior to the examination.

\section{Patients}

Patients were only included in the study if they fulfilled the following criteria: (1) referral for clinical body MRI (i.e. MRI of the chest, abdomen, pelvis or musculoskeletal system, including MRI of the spine) or referral for MRI angiography of the thoracic, abdominal or pelvic vessels, as well as of the peripheral arteries; (2) necessity to perform a medicamentous sedation prior to MRI, based on the request of the patient or the referring physician due to claustrophobia of the patient. Exclusion criteria were: age $<18$ years, general contraindications for MRI (i.e. cardiac pacemakers, neurostimulators, ferromagnetic implants etc.), pregnancy, drug or ethanol abuse, breast feeding, general contraindications for the use of midazolam (i.e. myasthenia gravis, known reverse or allergic reactions etc.), participation in another study simultaneously, and the presence of otorhinolaryngeal diseases (e.g. status postsurgery, rhinitis, nasal polyposis).

For this study, a total of 72 consecutive adult patients ( 42 females, 30 males, mean age 52.7 years; total of 72 scheduled examinations) were included. These patients were randomly assigned to one of two treatment groups (TG) according to a randomisation list. Patients in TG1 received $7.5 \mathrm{mg}$ midazolam orally (Dormicum, Roche Pharma AG, Reinach, Switzerland) $15 \mathrm{~min}$ before MRI. Patients of TG2 received one pump corresponding to $0.1 \mathrm{ml}$ of a ready-to-use nasal spray containing a $0.5 \%$ midazolam solution into each nostril immediately prior to MRI (in total, $1 \mathrm{mg}$ midazolam). If the dose of one pump per nostril was not sufficient in order to perform MRI, the intranasal administered dose could be increased to up to two pumps per nostril (total of $2 \mathrm{mg}$ midazolam). The decision on whether a second intranasal dose of midazolam had to be applicated was made by the attending radiologist.
The intranasal midazolam formulation was developed especially for this purpose according to the NRF recommendations (Neues Rezeptar Formularium, ABDA Bundesvereinigung Deutscher Apothekerverbände, D65760 Eschborn, Govi-Verlag Pharmazeutischer Verlag $\mathrm{GmbH}, 1999$ ) and manufactured by a pharmacist (J.M.F.). The ready-to-use nose spray in the off-label use contained $3 \mathrm{ml}$ of the injectable aqueous $0.5 \%$ Dormicum solution (Roche Pharma AG, Switzerland) with $0.5 \mathrm{mg}$ midazolam $(0.1 \mathrm{ml})$ per single dose. For conservation, $10 \mathrm{mg} / \mathrm{ml}$ benzyl alcohol were added. The solution was filtered through a $0.22-\mu \mathrm{m}$ sterile filter (OptiScale, Millipore Corporate, Billerica, MA, USA) and prepared under aseptic conditions in order to reduce, as far as possible, any bacterial contamination and possible glass splinters resulting from the opening of the glass ampoules. The device for application of the nose spray consisted of a 10-ml bottle with a microdoseur nose adapter (Pharminnova, Waregem, Belgium) screwed on top of it, allowing a reproducible and standardised dosing of $0.1 \mathrm{ml}$ (Fig. 1). In order to exclude the administration of air, it was important to use this type of device in an upright position of the patient after having checked the complete filling of the tubes and pump system by spraying once into the air (Fig. 2).

\section{Monitoring of patients during MRI}

Each patient received a venous cannulation before the application of midazolam for safety reasons in order to assure venous access in case of adverse effects. The antagonist flumazenil (Anexate, Roche Pharma AG, Switzerland) was available at any time. For safety reasons,

Fig. 1 Photograph of the used ready-to-use nasal spray for the midazolam application. The device for application of the ready-to-use nasal spray consisted of a $10-\mathrm{ml}$ bottle with a microdoseur nose adapter screwed on top of it, allowing a reproducible and standardised dosing of $0.5 \mathrm{mg}$ per pump

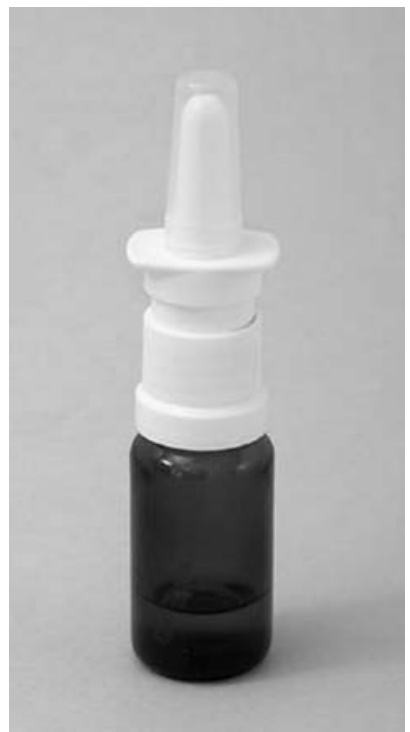


Fig. 2 Photograph shows how the ready-to-use nasal spray was administered. In order to exclude the administration of air, it is important to use this type of device with the patient being in an upright position

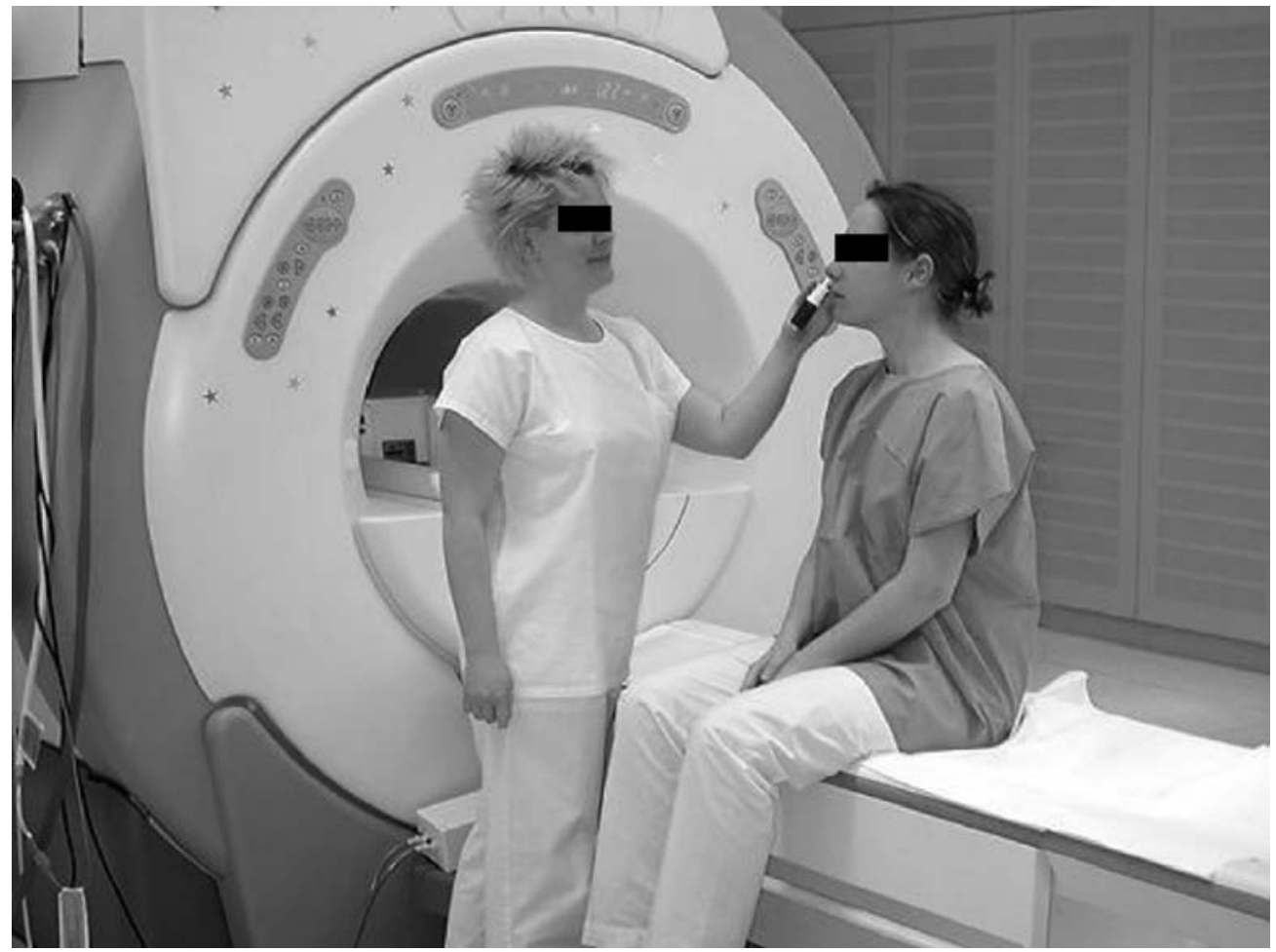

all patients were warned to not drive a vehicle or to operate machinery 24 hours after the application of midazolam. During MRI, patients were in permanent contact with the investigating technician by means of headsets and a microphone. Heart rate and respiratory frequency were surveyed during the whole examination. Additionally, the patient received an emergency ball to disrupt the examination if necessary.

\section{MRI}

MRI was performed on one of two 1.5-T scanners (Signa EchoSpeed Plus, Signa TwinSpeed Plus; GE HealthCare, WI, USA) according to the corresponding examination protocol (Table 1). All MRI protocols were standardised and consisted of various sequences without and with a contrast agent. The number of coils as well as the patients' position were selected depending on the investigated body region. If necessary (particularly concerning abdominal and thoracic MRI), special examination techniques were used, such as respiratory triggering and breath-holding techniques.
Data analysis

\section{Feasibility of the study}

In both TGs, the examination was classified as "feasible" or "non-feasible." The study was categorised as "feasible" if all of the planned MRI sequences could be accomplished. If one or more MRI sequences of the corresponding protocol could not be performed, the study was rated as "not-feasible" and the reason for the termination of the examination was noted.

\section{Anxiety, sedation and adverse effects}

The efficiency of midazolam in both TGs was assessed by the patients themselves, as well as by the technician who performed the MRI examination. Patients' anxiety before the examination, as well as the experienced fear during the investigation, was evaluated by a visual analogue scale (VAS) immediately before and $15 \mathrm{~min}$ after MRI. The VAS was presented as a line of defined length (commonly, $100 \mathrm{~mm}$ ) with anchors at either end. The patients were instructed to grade their fear sensation by placing a mark between the two anchors, without being told the precise distance between them. The left anchor was defined as the absence of fear, the right anchor was defined as maximal fear. The distance of one of the anchors and the patient's 
Table 1 Overview of the performed magnetic resonance imaging (MRI) protocols
$\mathrm{TG}=$ treatment group; $\mathrm{SD}=$ standard deviation; ${ }^{1}=$ number of examinations; ${ }^{2}=$ nine examinations in TG1 could not be started due to claustrophobia

\begin{tabular}{|c|c|c|c|c|c|}
\hline \multicolumn{2}{|c|}{ MRI protocol } & \multicolumn{2}{|c|}{ TG1 } & \multicolumn{2}{|c|}{ TG2 } \\
\hline Neck & & $\begin{array}{l}n^{1} \\
2\end{array}$ & $\frac{\text { Mean scanning time }(\min )}{43}$ & $\begin{array}{l}n^{1} \\
2\end{array}$ & $\frac{\text { Mean scanning time }(\mathrm{min})}{30}$ \\
\hline Thorax & Thoracic outlet & 2 & 33 & 2 & 43 \\
\hline \multirow[t]{4}{*}{ Abdomen } & Liver & 3 & 48 & 5 & 52 \\
\hline & Pancreas & 1 & 45 & 1 & 45 \\
\hline & Kidney/adrenals & 1 & 40 & 1 & 45 \\
\hline & Defecography & 0 & - & 1 & 20 \\
\hline Pelvis & & 4 & 32 & 5 & 45 \\
\hline \multirow[t]{4}{*}{ Spine } & Cervical & 2 & 28 & 2 & 28 \\
\hline & Thoraic & 1 & 30 & 0 & - \\
\hline & Lumbar & 3 & 28 & 3 & 34 \\
\hline & Lumbar incl. sacroiliac joints & 0 & - & 2 & 35 \\
\hline \multirow[t]{4}{*}{ Extremity } & Shoulder & 2 & 42 & 4 & 41 \\
\hline & Forearm & 1 & 15 & 1 & 25 \\
\hline & Knee & 2 & 40 & 1 & 30 \\
\hline & Forefoot & 1 & 45 & 2 & 40 \\
\hline \multirow[t]{3}{*}{ MRA } & Thoracic & 1 & 40 & 1 & 35 \\
\hline & Abdominal & 1 & 30 & 2 & 38 \\
\hline & Pelvic/femoral & 0 & - & 1 & 40 \\
\hline \multicolumn{2}{|c|}{ Total/mean $(+/-S D)$} & $27^{2}$ & $36.0(+/-16.8)$ & 36 & $39.4(+/-15.5)$ \\
\hline
\end{tabular}

mark was expressed in millimetres of the maximum of fear $(100 \mathrm{~mm})$. A reduction of the anxiety on the VAS below $50 \%$ was categorised as "not sufficient," and over $70 \%$ was categorised as "sufficient." Values in between were described as "non-classifiable." In addition, all patients received two questionnaires. One of these questionnaires was filled out at our institution about 15 min after MRI. The patients were asked if they had faced problems during the examination (e.g. recurrent fear sensations), of what kind these problems had been and if they had experienced any adverse effects by the use of the midazolam spray (such as nasal burning sensation, pain etc.). Additionally, the patients had to answer the question as to whether they would repeat the examination. Finally, the patients were asked to fill out a second questionnaire at least five days after MRI and to send it back to our institution. In this second questionnaire, the patients could note for how long their attention was reduced, whether problems or adverse effects arose after leaving the radiology department and, again, whether they would repeat the MRI procedure. The technician who performed MRI was also asked to fill out a standardised questionnaire for each patient. This questionnaire contained several questions concerning the patient's cooperation and a subjective impression of the degree of anxiety or sedation of the patient during the examination.

\section{MRI image quality}

Image quality among the patients of TG1 and TG2 was rated by a single radiologist (D.W.) with 10 -years of experience in body MRI. The reader was blinded concerning the application form of midazolam (oral versus intranasal). MRI image quality of each MRI examination was assessed using the following five-grade scale: grade 0 , very poor image quality; grade 1, poor image quality; grade 2 , satisfactory image quality; grade 3 , good image quality, and grade 4, excellent image quality. Grade 0 or 1 was applied if the examination was of no or very little diagnostic usefulness because of extensive motion artifacts that were not caused by pulsation or normal peristalsis. Examinations classified as grade 2 allowed us to make the diagnosis, but some motion artifacts were still present. Examinations graded as 3 and 4 included a good or excellent image quality, with no or almost absent motion artifacts.

\section{Statistics}

Statistical analysis was performed by SPSS 12.0.1 for Windows (SPSS Inc.; Chicago, IL, USA). Statistical significance was accepted if $p<0.05$. For MRI image quality, the Chi-square test was used. The values of the VAS in both TGs were compared by means of the MannWhitney test. Analysis of the questionnaire concerning the willingness to repeat the MRI examination was made by Fisher's exact test.

\section{Results}

Seventy-two patients were scheduled for a total of 72 MRI examinations (Table 1 ). There were no statistically signif- 
icant differences between both TGs with regards to the examined body regions and examination techniques [examinations with respiratory triggering: 8 (TG1)/10 (TG2); examinations with breath-hold technique: 11 (TG1)/13 (TG2)]. There were also no statistically significant differences with regards to gender, age and weight of the patients (female/male ratio in TG1 19/17, in TG2 23/13; mean age $+/-\mathrm{SD}$ in TG1 51.8 years $+/-10.9$, in TG2 53.6 years $+/-12.6$; mean weight $+/-$ SD in TG1 $81 \mathrm{~kg}+/$ -12.9 , in TG2 $75 \mathrm{~kg}+/-17.8$ ).

\section{Feasibility of the study}

After oral application of midazolam, MRI had to be cancelled in 9/36 examinations $(25 \%)$ due to patients reasons. MRI could not be started in another nine patients $(25 \%)$ due to insufficient sedation of the patient, resulting in $18 / 36$ non-diagnostic studies $(50 \%)$. There were no cancellations of MRI after the application of intranasal midazolam. Only one patient $(2.8 \%)$ in TG2 had a nondiagnostic examination, since he could not cooperate due to amblyacousia (Fig. 3).

Vital signs and adverse effects

There were no significant changes in the heart rate or respiratory frequency before, during and after the examination in either TG. The antagonist flumazenil (Anexate, Roche Pharma AG, Switzerland) was not needed in any case. No patient required special monitoring or treatment after the examination. Apart from a temporary nasal burning sensation in $61.1 \%$ (22/36 patients) of the patients of TG2, there were no relevant adverse effects in both TGs.

Anxiety and degree of sedation

The evaluation of the VAS for the reduction of the pain experienced resulted in a highly significant difference between the two TGs $(p<0.001)$. In TG1, the reduction of anxiety was rated as not sufficient (i.e. a decrease of the anxiety as assessed on the VAS below $50 \%$ ) by $30 / 36$ patients $(83.3 \%)$, whereas the patients of TG2 showed a sufficient reduction of anxiety (i.e. a decrease of the anxiety as assessed on the VAS over $70 \%$ ) in $35 / 36$ patients $(97.2 \%)$ (Fig. 3). The dose of $1 \mathrm{mg}$ in TG2 was sufficient in 31 patients, whereas the dose had to be repeated during the examination (total dose of $2 \mathrm{mg}$ ) in five patients $(13.9 \%$ ).

According to the questionnaires which were filled out directly following MRI, 18/36 (50\%) patients of TG1 would repeat the examination, $3 / 36$ patients would not repeat the examination and 15/36 patients did not mark an answer. Twenty of thirty-six (20/36) patients of TG2 would repeat the examination and $3 / 36$ patients denied to a repeat of the examination or to undergo MRI in future. Concerning the questionnaires which were filled out by patients at home, we only received 11/36 (30.6\%) letters from patients after spray application, as well as 6/36 (16.7\%) letters from patients after the oral application of midazolam. Therefore, no statistical evaluation was possible. All 11 patients who received the midazolam spray and who had sent back the questionnaire reported that they would repeat the investigation $(100 \%)$, whereas only $3 / 6$ patients of TG1 $(50 \%)$ returning the questionnaires would repeat the MRI proce-
Fig. 3 Diagram illustrating the number of patients in both treatment groups (TGs) with a feasible MRI examination, sufficient anxiety reduction $(>70 \%$ measured by visual analogue scale, VAS) and satisfactory to excellent MRI image quality (grades 2 to 4 )

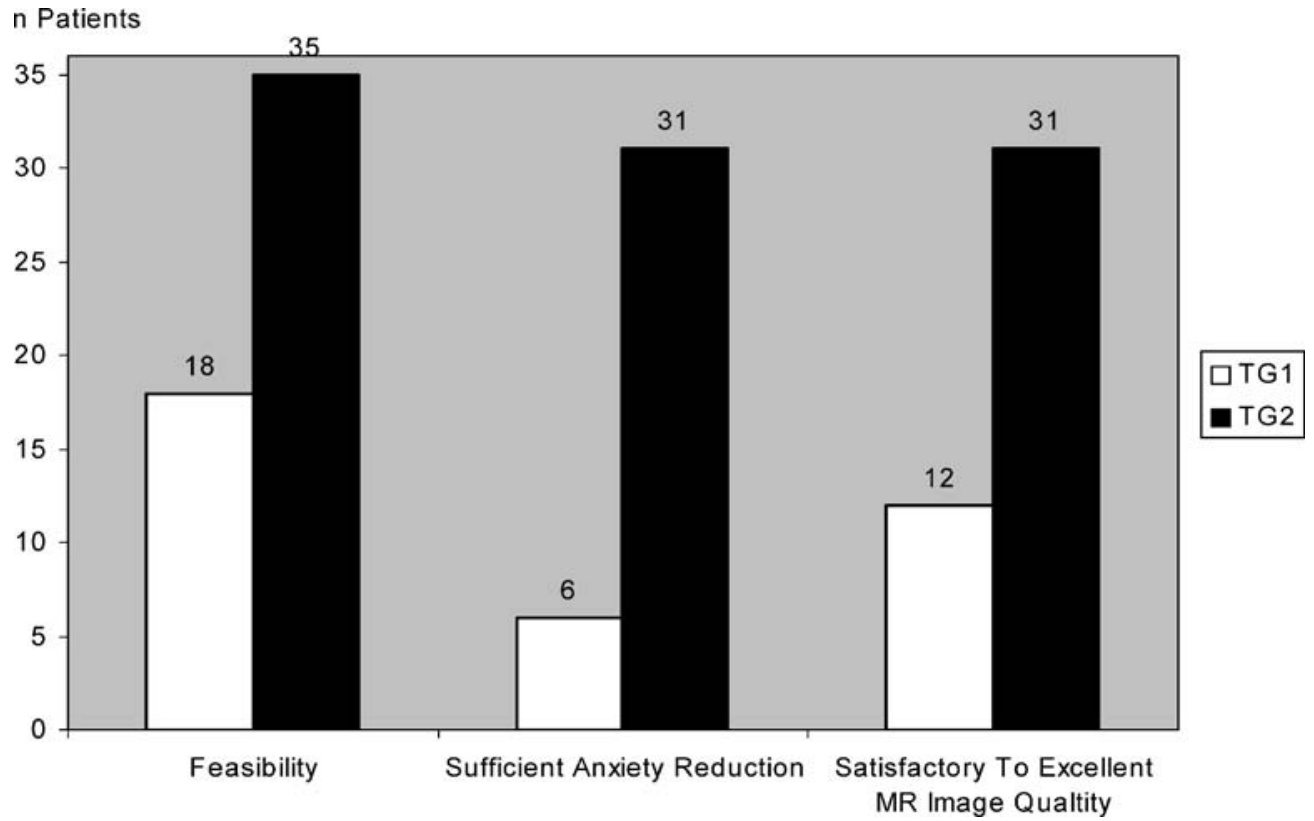


dure. The duration of attention restriction was stated with a high variability by the patients. The data varied between 0 and $120 \mathrm{~min}$ (mean $19.8 \mathrm{~min}$ ) after intranasal application and 15 to $120 \mathrm{~min}$ (mean $67.5 \mathrm{~min}$ ) after oral medication. These values did not differ statistically significantly $(p=0.275)$. The technician's impression about patient anxiety and cooperation during MRI was significantly better in TG2 versus TG1 $(p<0.001)$. In 34/36 examinations $(94.4 \%)$ of TG2, the technician had a positive impression, whereas in TG1, the technician's impression was only positive in $9 / 36$ examinations $(25.0 \%)$.

\section{MRI image quality}

In contrast to oral application (12/36), the image quality after intranasal application of only $1 \mathrm{mg}$ midazolam was satisfactory to excellent in most MRI examinations (31/36), independent from respiratory triggering, as well as breathhold techniques (Fig. 3). Statistical evaluation of the image quality resulted in a highly significant difference in favour of TG2 $(p<0.001)$.

\section{Discussion}

Conscious sedation is one of the most important measures to help claustrophobic patients to cope with MRI examination. The term "conscious sedation" means that the patient is able to maintain his/her airway and respond appropriately to physical stimulation and verbal commands while sedated [10]. For this purpose, midazolam is often used because its pharmacological properties are superior to other benzodiazepines (fast onset, better tissue compatibility, controllability of effect, short duration of action of 20 to $40 \mathrm{~min}$, short elimination half-time of 1.5 to $3 \mathrm{~h}$ ). Additionally, anxiolysis appears already at a low dosage and, usually, there are no relevant adverse effects, apart from a slight sedation [3].

Midazolam may be administrated orally, intravenously, intrarectally or intranasally. For radiological examinations, in particular, to achieve conscious sedation in claustrophobic patients referred for MRI, midazolam is generally administrated via the oral or the i.v. route in most institutions. The advantage of oral administration compared to i.v. administration is the fact that it does not present an invasive procedure and most radiologists feel more comfortable with oral use than with i.v. use. On the other hand, i.v. use compared to oral application offers the advantage that there is no hepatic first-pass metabolism, resulting in a faster onset of action and a higher bioavailability of the drug. In addition, i.v. administration is not influenced by the bowel contents and movement, resulting in an improved controllability $[11,12]$.

In the present clinical trial, we compared $7.5-\mathrm{mg}$ midazolam tablets with intranasal low-dose application.
This oral dose has been described to offer a favourable relationship between desirable and undesirable effects [13]. The inferior result of the oral application regarding feasibility of the examinations, reduction of anxiety and image quality might be explained by the study of Biro et al. [13]. Biro et al. [13] found a marked interindividual variability of sedation and amnesia after orally administered midazolam prior to surgery. Additionally, anxiolysis was lacking in all orally administered doses, showing no difference compared to the placebo group, whereas sedation and amnesia were dose-dependent.

The intranasal application of midazolam is well known in paediatric medicine, especially for painful procedures such as dental interventions, treatment of acute seizures, for premedication and trauma management $[5,6]$. In adults, the intranasal application of midazolam has been reported for sedation during upper gastrointestinal endoscopy, for premedication, as well as for the treatment of panic disorders [3, 7, 8]. However, the experience with midazolam in patients for radiological examinations and, in particular, in patients referred for MRI is limited. Several years ago, Moss et al. [14] presented their preliminary results using intranasal midazolam for claustrophobic MRI patients. This route of administration reduced the necessity for i.v. sedation from $67 \%$ to $17 \%$. Recently, Hollenhorst et al. [2] showed, in a double-blind placebo controlled study, that intranasally applicated midazolam leads to a significant reduction in MRI-related anxiety, resulting in an improved MRI image quality. In the study of Hollenhorst et al. [2], 54 consecutive patients scheduled for MRI of the head were divided into two groups receiving either $4 \mathrm{mg}$ of midazolam intranasally or a placebo intranasally. No cancellation of MRI occurred in the midazolam group consisting of 27 patients, whereas $4 / 27(14.8 \%)$ patients receiving the placebo panicked and terminated MRI earlier. Our study differs from that of Hollenhorst et al. [2] in many respects. In contrast to Hollenhorst et al. [2], we only administered $1 \mathrm{mg}$ of midazolam intranasally in the majority of cases. The effectiveness of the relatively low dose of $1 \mathrm{mg}$ of midazolam is known from other applications. Schweizer et al. [3] reported an improvement of panic disorders in $4 / 5$ patients using a total dose of $0.5-$ $1.0 \mathrm{mg}$ of self-administrated intranasal midazolam drops. Moss et al. [14] also used a total dose of $0.5-1.0 \mathrm{mg}$ in claustrophobic patients scheduled for MRI, reducing dramatically the number of patients needing additional i.v. sedation. Another difference to the study of Hollenhorst et al. [2] is the time delay between the application of the drug and the start of MRI. In their study, intranasal application of midazolam took place 15 min before MRI. In the study of Moss et al. [14], patients received two drops per nostril of a midazolam solution (total $0.5 \mathrm{mg}$ ) prior to entering the scanner without a time definition. This dose could be repeated during the MRI procedures (total $1.0 \mathrm{mg}$ ).

In our study, we administered the intranasal spray just before MRI. According to the literature, the effect of the 
intranasally applicated midazolam starts in less than $5 \mathrm{~min}$ [10]. In a clinical setting, it is easier to administer the intranasal spray on the MRI table if the patient is complaining of claustrophobia. Moreover, one main difference compared to the study of Hollenhorst et al. [2] is that our patients were scheduled for body MRI and not for a neuroradiological indication. In the study of Moss et al. [14], the kind of examined body regions was not reported. Compared to neuroradiological MRI, body MRI is usually more time consuming and partially requires particular techniques which need the compliance and active cooperation of the patient.

The excellent effect of the intranasally used low-dose midazolam may be explained by the following reasons. In contrast to oral and rectal administration, intranasal midazolam administration has no first-pass effect in the liver and no interference with the bowel contents. Therefore, the interindividual differences in the dose-related effects are much smaller. Using a nasal spray absorption through the nasal mucosa is fast and virtually complete (about $83 \%$ ), resulting in a quite easily controllable midazolam application [15]. The fast and intense effect of the small and amphiphilic midazolam molecule may be explained by the supposed predominant fast paracellular transport through the nasal mucosa, as well as the easy passage through the blood-brain barrier [16, 17]. According to the literature, the intranasal spray is also superior to nose drops, which are partially swallowed [2, 8, 13]; compared to i.v. administration, there is a lower toxicity due to decreased peak concentration and its usage is easier and less painful $[2,15]$.

Although intranasal application is an off-label use and not yet approved by the authorities, it seems to be safe. As also described by other authors, there were no major adverse effects requiring further therapy, only a local temporary burning sensation after intranasal spray applica- tion was detected in $61.1 \%(22 / 36)$ of patients $[2,14]$. This is probably due to the used preserving agent (benzyl alcohol) and the low $\mathrm{pH}$ value (3.3) of the aqueous midazolam solution [15]. Before starting a broad clinical use of the low-dose intranasal application, further efforts should be made to eliminate this burning sensation, by using another preserving agent for example. Additionally, a spray form applicable in a lying position is desirable, as MRI patients often are lying on the examination table when mentioning claustrophobia. This could be the subject of future investigations.

We acknowledge the following limitations. In this study, we only used a VAS to quantify the severity of claustrophobia before and after MRI; no psychometric test was made, especially no Spielberger State-Trait Anxiety Inventory (STAI). The reason for this is that Dantendorfer et al. [18] could not find a correlation between the values of STAI and the presence of motion artifacts. They assumed that STAI is not able to measure the "special fear" caused by the MRI scanner. Probably, the VAS just before and after MRI represents more accurately the reality. On the other hand, Hollenhorst et al. [2] found similar results using both STAI and VAS. Hence, it may be concluded that the VAS is probably sufficient to assess the grade of anxiety. Other investigators also worked successfully using only the VAS [13]. Additionally, the time delay of 15 min between the oral administration of midazolam and MRI could be too short, possibly leading to the bad results in TG1. In other studies, a longer time interval (e.g. $60 \mathrm{~min}$ ) has been used [13].

In conclusion, the intranasal application of low-dose midazolam immediately before MRI is an effective and patient-friendly solution to overcome anxiety in claustrophobic patients and results in a good image quality and a higher rate of feasible examinations.

\section{References}

1. Melendez JC, McCrank E (1993) Anxiety-related reactions associated with magnetic resonance imaging examinations. JAMA 270(6):745-747

2. Hollenhorst J, Munte S, Friedrich L, Heine J, Leuwer M, Becker H, Piepenbrock S (2001) Using intranasal midazolam spray to prevent claustrophobia induced by MR imaging. AJR Am J Roentgenol 176(4):865-868

3. Schweizer E, Clary C, Dever AI, Mandos LA (1992) The use of lowdose intranasal midazolam to treat panic disorder: a pilot study. J Clin Psychiatry 53(1):19-22
4. Walbergh EJ, Wills RJ, Eckhert J (1991) Plasma concentrations of midazolam in children following intranasal administration. Anesthesiology 74(2):233-235

5. Harbord MG, Kyrkou NE, Kyrkou MR, Kay D, Coulthard KP (2004) Use of intranasal midazolam to treat acute seizures in paediatric community settings. J Paediatr Child Health 40(910):556-558

6. Lloyd CJ, Alredy T, Lowry JC (2000) Intranasal midazolam as an alternative to general anaesthesia in the management of children with oral and maxillofacial trauma. Br J Oral Maxillofac Surg 38(6):593-595
7. Uygur-Bayramicli O, Dabak R, Kuzucuoglu T, Kavakli B (2002) Sedation with intranasal midazolam in adults undergoing upper gastrointestinal endoscopy. J Clin Gastroenterol 35 (2):133-137

8. Bjorkman S, Rigemar G, Idvall J (1997) Pharmacokinetics of midazolam given as an intranasal spray to adult surgical patients. Br J Anaesth 79 (5):575-580

9. Burstein AH, Modica R, Hatton M, Forrest A, Gengo FM (1997) Pharmacokinetics and pharmacodynamics of midazolam after intranasal administration. J Clin Pharmacol 37(8):711-718 
10. Merrick PA, Ramsby GR (1994) Conscious sedation for imaging and interventional studies. Radiol Manage 16 (2):35-40

11. Nordt SP, Clark RF (1997) Midazolam: a review of therapeutic uses and toxicity. J Emerg Med 15(3):357-365

12. Reed MD, Rodarte A, Blumer JL, Khoo KC, Akbari B, Pou S, Kearns GL; Pediatric Pharmacology Research Unit Network (2001) The single-dose pharmacokinetics of midazolam and its primary metabolite in pediatric patients after oral and intravenous administration. J Clin Pharmacol 41(12):13591369
13. Biro P, Weidmann G, Pietzsch S, Alon E, Brugger P (1997) [The dose-dependent effects of oral premedication with midazolam]. Anasthesiol Intensivmed Notfallmed Schmerzther 32(11):672677

14. Moss ML, Buongiorno PA, Clancy VA (1993) Intranasal midazolam for claustrophobia in MRI. J Comput Assist Tomogr 17(6):991-992

15. Knoester PD, Jonker DM, van der Hoeven RTM, Venneij TAC, Edelbrock PM, Brekelmans GJ, de Haan GJ (2002) Pharmacokinetics and pharmacodynamics of midazolam administered as a concentrated intranasal spray. A study in healthy volunteers. Br J Clin Pharmacol 53(5):501-507

16. Merkus F, Lehr C (2004) Intranasale Applikation von Arzneistoffen mit systemischer Wirkung. Deutsche Apotheker Zeitung 144(4):61-65
17. Fischer S, Renz D, Kleinstuck J, Schaper W, Karliczek GF (2004) [In vitro effects of anaesthetic agents on the blood-brain barrier]. Anaesthesist 53(12):1177-1184

18. Dantendorfer K, Amering M, Bankier A, Helbich T, Prayer D, Youssefzadeh $\mathrm{S}$, Alexandrowicz R, Imhof $\mathrm{H}$, Katschnig H (1997) A study of the effects of patient anxiety, perceptions and equipment on motion artifacts in magnetic resonance imaging. Magn Reson Imaging 15(3):301-306 